\title{
Survey Mode as a Moderator of Context Effects
}

\author{
May Doušak ${ }^{1}$
}

\begin{abstract}
After decades of survey quality research and many experiments on the mode of data collection as a source of data inequivalence, there is still no exact explanation of how the mixed-mode design impacts the collected data. While not providing a definitive answer, we propose a new explanation of the mode effect and how it correlates with other sources of errors in surveys. By reviewing the relevant literature, we develop theoretical arguments indicating that the mode of data collection is in fact a moderator of context effects, a view that can provide an answer to the question concerning the origin of the data cacophony in mixed-mode comparative studies.
\end{abstract}

\section{Introduction}

Research on the comparability of data obtained using different data collection modes in surveys is often associated with recent developments in quantitative social research. The falling response rates in traditional self-administered paper, face-to-face and telephone surveys together with the ever greater time and budget constraints create challenges for the traditional survey approach. As a result and given the many possibilities digitalisation offers, it is not uncommon for traditional data collection modes in both longitudinal and cross-sectional studies to be complemented with data collected through the Internet. After all, this is a way of reducing both the cost and duration of the survey fieldwork process (Dillman and Christian, 2005; Jäckle, Roberts, and Lynn, 2010).

Yet, in reality, these are not new developments. Market research agencies have been mixing modes of data collection since the 1930s after discovering that telephone surveys provide a faster and more cost-effective way of obtaining data in larger American towns (Karol, 1937). In 1944, Deming factored "Different kinds and degrees of canvas" as one of the sources of errors in surveys. He also noted that "cheaper methods" such as telephone (nowadays the Web) may hold greater utility once the comparability of data collection modes is obtained through more extensive research (Deming, 1944). Regarding research on the comparability of data obtained using different modes, already in 1963 Cannell and Fowler developed a set of hypotheses on how the interviewer's presence affects a respondent's motivation and on the underreporting of potentially "embarrassing" data (Cannell and Fowler, 1963).

Seventy years after Deming's article, great progress has been made on systematising measurement errors in different data collection modes (de Leeuw and Berzelak in Wolf et al, 2016; Berzelak, 2014; Vannieuwunhuyze, Loosveldt, and Molenberghs, 2010;

\footnotetext{
${ }^{1}$ Faculty of Social Sciences, University of Ljubljana, Ljubljana, Slovenia; may.dousak@fdv.uni-lj.si
} 
Roberts, 2007), although there is still a general consensus among the research community that the different modes do not provide equivalent data and therefore combining modes should be avoided where possible (Martin and Lynn, 2011). ${ }^{2}$

In addition, the advent of connected portable devices such as smartphones and wearables that store vast amounts of users' information online and give researchers an opportunity for continuous "big data" collection raise questions about the need for survey modes which would present an alternative to costly and lengthy face-to-face or lately even mailed paper and pencil questionnaires (Mayer-Schönberger and Cukier, 2013). Combined with time and budget constraints, respondents' reluctance to participate along with greater competiveness from private marketing and survey agencies, this forces researchers into streamlining survey designs and seeking new approaches to collecting data.

This explains why, in practice, the choice of a combination of data collection modes or new approaches is sometimes all that is available to researchers in order to continue with their research. To ensure high quality data, researchers must carefully assess all possible measurement specifics of different data collection modes and sources of bias (Jäckle, Roberts, and Lynn, 2010). For this purpose, we propose a new view on the above phenomenon. Based on evidence stemming from both theoretical and empirical sources, we conceptually discuss the thesis that the mode of data collection moderates the context effects.

\section{About Context Effects}

Before digging deeper into mode effects and their relationship with context effects, we consider different authors' views on context effects by summarising certain key views on context effects found in the literature. ${ }^{3}$

Social methodologists typically refer to them as intra-survey stimuli that can have a noticeable effect on the measurement (Schwarz and Strack, 1991). The most evident is the context given in the question wording: asking about one's attitude to abortion in the context of saving a mother's life might bring different responses to the same question in the context of unwanted children (Tourangeau and Rasinski, 1988).

In addition to this more obvious example, various experiments show that prior questions or topics can also influence responses to later questions (a phenomenon called the question-order effect) (Hafner-Fink and Uhan, 2013). Tourangeau and Rasinski's (1988) four-stage response process in attitude measurement clearly explains this bias caused by prior topics in a survey. According to their definition, respondents:

1. "first interpret the attitude question, determining what attitude the question is about",

2. "retrieve relevant beliefs and feelings",

3. "apply these beliefs and feelings in rendering the appropriate judgement"

\footnotetext{
${ }^{2}$ While this is true for most surveys, researchers sometimes combine the interviewer-administered data collection mode (e.g. CAPI) with the self-administered mode for part of the questionnaire (e.g. drop-off questionnaire, CASI) for sensitive topics where the interviewer's involvement might have an influence on the respondent (de Leeuw, 2005).

${ }^{3} \mathrm{~A}$ discussion of all of the context effects, their causes and consequences lies beyond the scope of this article; we only refer to some of the context effects needed to convey our message.
} 
4. "use this judgement to select a response" (Tourangeau and Rasinski, 1988: 300).

If the "attitude" and thus also "relevant beliefs and feelings" have already been determined and retrieved by prior items in a survey, they could be retrieved in the context of something which may have a great influence on their judgement process. Tourangeau et al. (2000) later expanded the four-stage response process beyond attitude measurement only, although they still discussed the attitudinal questions in a separate section due to their greater measurement instability. There they explicitly noted "minor question wording or order" (Tourangeau et al., 2000:13) as one of the reasons for shifts in measurement. While there is no reason not to apply such ideas to factual types of questions, here we limit the discussion solely to attitudinal questions.

The example of a question on one's attitude to abortion could also have an impact on more general questions later in the survey as the term "abortion" has already been put into context. Schuman and Presser did a split-ballot experiment on this very topic and demonstrated a $13 \%$ point difference in answering "yes" to the same question "Do you think it should be possible for a pregnant woman to obtain a legal abortion if she is married and does not want any more children?" due to the question-order effect (Schuman and Presser, 1996).

Holyk also explained context effect in terms of priming as psychologists refer to it (Holyk in Lavrakas, 2008). By priming, the words that are semantically or associatively related to prior terms in a questionnaire are recognised faster than other words. To give an example, if "doctor" is mentioned in previous questions, the word "nurse" is recognised faster. In this case, interpretation and determination of what the question is about occur more impulsively and may be given less thought and judgement. For some authors, especially social methodologists, context effects come only from within the questionnaire itself (intra-survey stimuli). Besides question wording, question order and priming mentioned above, intra-survey stimuli include (but are not limited to) response scale selection, serial position effect (primacy, recency), and others (Lavrakas, 2008).

In contrast, Bradburn (1991) pointed out that context effects consist of much more than just question-order effects or even the questionnaire itself. He argued that "everything that occurs during an interview has the potential to influence the answers given by respondents to the questions asked by the interviewer" - a view that also accounts for extra-survey stimuli (Bradburn, 1991). While some readers might argue that his choice of wording implies the presence of the interviewer, the author also discusses mail questionnaires. By explicitly mentioning nonsemantic information during an interview such as "the social context within which the interview takes place", Bradburn takes a broader, psychologist's viewpoint on the survey context. Due to such different views on the context of survey interviews, Schwarz and Sudman even contended that cognitive researchers and social methodologists do not speak the same language (Schwarz and Sudman, 1991).

Given that the most commonly recognised model for the cognitive processes underlying a survey conversation (Tourangeau, Rips and Rasinski, 2000) as well as its descendant model of satisficing (Krosnick, 1991) both come from psychologists, we suggest taking a wider view of context effects in surveys, similarly to Callegaro, Lozar Manfreda and Vehovar (2015).

Under this broader view, stimuli can also come from outside the survey questionnaire itself (extra-survey context effects). Examples of such stimuli include (among others) 
time constraint and external distractions such as looking out for children or driving a car while responding to an interview as the respondent is not completely engaged in the survey response process. According to this view, even the current political situation and the respondent's cultural background may have an influence on their responses. The presence of other people can also considerably influence responses to questions on sensitive topics - imagine asking a teenager about their experience with marijuana in the presence of their parents. When talking about the presence of other people, social conformity should be mentioned (Kelman, 1958). Asking about one's attitude to abortion at a Christian gathering would see results as predicted. Even the interviewer's presence can impact responses such that the respondent over-reports socially acceptable behaviour and under-reports less socially acceptable behaviour, what is called "social desirability bias" (Roxas and Lindsay, 2012; Chang and Krosnick, 2010; Sakshaug, Yan, and Tourangeau, 2010; Tourangeau and Yan, 2007). In order to reduce the cognitive burden of providing accurate and consistent answers, the respondent might also answer in terms of satisficing the interviewer (Krosnick, 1999).

The interviewer, specifically, can also be regarded as a source of context effect. Namely, an important aspect of the interviewer's presence is their conversational implicatures ${ }^{4}$ (Grice, 1974). Without an interviewer, the respondent hears (pre-recorded) or only reads the question literally as it is written. While the interviewer should read the questions literally as they are written in the questionnaire, he or she does indeed have an influence on the flow of conversation. Grice (1974) states that dialogues should comply with four maxims:

- quantity (be as informative as required - but not more)

- quality (do not spread untrue or unconfirmed information)

- relation (be relevant)

- manner (it is not about the content but about the way the information is given in terms of ambiguities, obscurities, prolixity etc.) (Grice, 1974, p. 47).

According to these four maxims, the conversation can indeed change when an interviewer is present compared to self-interviewing (the so-called interviewer effect). Apart from semantic communication, the similarly important nonsemantic, tacit communication between the respondent and the interviewer should not be disregarded. Both Uhan and Bradburn used Grice's framework to argue that interviewer-respondent communication can be seen as a source of context effects (Bradburn, 1991; Uhan, 1998).

Another source of context effect can be stimuli that can influence a response if someone does not have first-hand experience on the topic - this fact is heavily relied upon in the media, marketing and politics to attract customers or win over sympathisers. One example is an individual's attitude to certain groups of people (based on religion, nationality, sexual orientation, sport club loyalty or other characteristics) they have never met but are given a negative connotation in media read by the respondent.

Uhan (1998) goes even further with his systematic distinction of "local" and "global" contexts. The local context encompasses the survey questionnaire and survey situation

\footnotetext{
${ }^{4}$ Indirectly, implicitly conveyed meaning in speech.
} 
which not only consist of wording and question-order effects, but also scale selection, content validity, the respondent's acquiescence, ${ }^{5}$ standardisation of procedures and relevant underlying psychological processes and respondent capability. The latter hints at both cognitive processes underlying survey interviewing (Tourangeau, Rips, and Rasinski, 2000) as well as the satisficing model (Krosnick, 1999). The idea of local context encompassing much more than the questionnaire itself is also supported by other authors who recognise the cognitive process model as capable of explaining "measurement errors due to respondents" (Callegaro, Lozar Manfreda, and Vehovar, 2015, p. 64).

The global context is defined as the specific environment of the interview, formed by the cultural as well as personality traits of both the interviewer and the respondent who define the interview's properties through the constellation of their relationship (Uhan, 1998). The author expands Bingham and More's (1934) view who state that a survey interview is a "conversation with a purpose", and Bradburn's (1991) view on context (the "social context within which the interview takes place") by suggesting that a survey interview is a specific social event.

This definition is particularly valuable while assessing the comparability of survey data between multiple global contexts among different cultural environments (cross-national surveys) or different target groups of respondents. Moreover, the global context hypothesis can also explain differences in data obtained from different data collection modes such as the interviewer effect as the interviewer brings his/her beliefs, personal traits, experience and knowledge into all of the interviews he/she conducts (Uhan, 1998). While one might argue that conversational implicatures should fall within the local context, Uhan explicitly placed them in the global context to emphasise the traits both the respondent and the interviewer bring into the survey situation.

Respondent motivation is another aspect of the context within which a survey interview is conducted. A respondent can be motivated by either the content of the survey (a close or salient topic) or its presentation (by the interviewer, visual appearance, technical form of the presentation) (Callegaro, Lozar Manfreda, and Vehovar, 2015; Dillman and Christian, 2005). Based on the above discussion, we systemise some of the selected context effects by positioning them within two categorisations arising from different authors' views: the view of the local and global context effects versus the view of intra-survey and extra-survey stimuli, as shown in Table 1.

In summary, we see context effects as arising not only from the survey questionnaire itself, but also from the survey situation, constellation of the interviewer and respondent, as well as their cultural, political and social backgrounds. This broad interpretation, which encompasses views held by both social methodologists and psychologists, provides the most suitable basis for our main argument, namely that survey mode moderates the context effect. To argue the relationship between the two, we will more specifically focus on extra-survey stimuli stemming from mode-inherent characteristics.

\footnotetext{
${ }^{5}$ Acquiescence can be defined as a personality trait (Couch and Keniston, 1960), as well as an effect of both survey content (Adorno, et al. 1950; Schuman and Presser, 1996) and survey situation (social context) (Campbell, et al., 1960). While Uhan (1998) explicitly placed it in local context in connection to the survey instrument, we refer to it in both the local and global contexts due to the different views on its cause and nature. The phenomenon has been studied thoroughly in psychological literature which the interested reader may consult (DeMaio, 1985; Adorno et al., 1950; Campbell, et al., 1960; Uhan, 1998).
} 
Table 1: Systematisation of global and local context effects versus intra-survey and extra-survey stimuli

\begin{tabular}{lll}
\hline & Intra-survey stimuli & Extra-survey stimuli \\
\hline Local context & - Question wording & - Time constraint \\
& - Question order, priming & - External distractions \\
& - Serial position effect (recency, & - Social conformity \\
& primacy) & - Acquiescence \\
- Acquiescence & - Respondent motivation \\
& - Respondent motivation & - Satisficing \\
- Scale selection & - Respondent capability \\
& - Standardisation of procedures & - Social context during inter- \\
& view \\
\hline Global context & - Acquiescence & - Interviewer's personality traits \\
& & and beliefs \\
& & - Conversational implicatures/ \\
& interviewer effect \\
& - Respondent's personality traits \\
& and beliefs \\
& - Cultural background (of re- \\
& spondent and interviewer) \\
& - Political and social context \\
\hline
\end{tabular}




\section{Mode Effects and their Relationship with Context Ef- fects}

The modes employed in collecting data in the measurement stage are constantly evolving, from paper and pencil, first documented in 1788, through face to face (1912), telephone (1930s) and more recently many other modes to web and mobile (Callegaro, Lozar Manfreda, and Vehovar, 2015; de Heer, de Leeuw, and van der Zouwen, 1999; de Leeuw, 1992). Not only are they constantly evolving, most data collection modes are here to stay since none outperforms the others in all respects (Couper, 2011). They can also be mixed in either a longitudinal or cross-sectional way, that is, when one mode is superseded by another in a long-running comparative survey, or when multiple data collection modes are used simultaneously, such as by the International Social Survey Programme (ISSP; ISSP, 2010).

The problem researchers face is that the different modes of data collection sometimes provide unequal data due to their specific mode-inherent characteristics. This data inequity phenomenon is called the mode effect. Abundant research and literature is available for the interested reader to consult ${ }^{6}$ on the modes of data collection, their main characteristics and the problem of combining them; however, the research on mode effects is lacking in its conclusions (de Leeuw, 2005).

For the purpose of this article, which seeks to relate mode effects to context effects, we shall explain the mode effect by narrowing our focus to some of the most commonly identified mode-inherent characteristics. But before that, let us raise attention to some effects which are commonly mistaken for mode effects and should be disregarded in our discussion.

When mixing data collection modes, researchers sometimes give respondents the freedom to choose their own preferred mode for participation. Self-administered paper-andpencil questionnaires can be combined with the Web by writing the URL of the Web questionnaire on top of page 1 of the questionnaire (Lozar Manfreda and Vehovar, 2002). As respondents select the mode of their participation based on their preferences, any differences between the modes in the resulting data should not be attributed to the mode effect only but to the selection effect as well. Differences in coverage and nonresponse of different modes also should not be mistaken for the effect of the data collection mode per se (Sax, Gilmartin, and Bryant, 2003; Dillman, et al., 2009; Kaplowitz, Hadlock, and Levine, 2004).

Another example of effect which can however not be regarded as a mode effect refers to the following situation. Survey design errors can remain hidden and undetected even after many years and waves of a survey. Sometimes, they are exposed by a change in the data collection mode. Dillman and Christian (2005) provide a vivid example of a survey which identified significantly more "divorced" and "widowed" and fewer "single" respondents after the data collection mode was switched from telephone to the Web. When looking into it, they found a huge shortcoming in the survey design: when the respondents had been asked about their marital status over the telephone, they were simply

\footnotetext{
${ }^{6}$ Examples include (among others) de Leeuw and Berzelak in Wolf et al, 2016; Callegaro, Lozar Manfreda, and Vehovar, 2015; Berzelak, 2014; Groves, et al., 2009; de Leeuw, Hox, and Dillman, 2008; Tourangeau, Rips, and Rasinski, 2000.
} 
asked about their status without being provided the full list of options. Many widowed or divorced respondents answered truthfully that they were single. In contrast, web respondents were given a full list of possible answers so they chose one of options provided. The inequivalence of the data collected in such cases is not mode-inherent as it could be avoided in the survey design (disregarding the recency effect we will mention later, the telephone question could be reformulated to "Now I will read some of the marital statuses (... ) Please choose the one that is nearest to your current marital status").

The above examples are not really mode-inherent effects and can (and therefore should) be reduced by the survey design (Hox, de Leeuw, and Zijlmans, 2015; Berzelak, 2014; de Leeuw, Hox, and Dillman, 2008). Thus, some of the most obvious causes of data inequivalence stemming from different data collection modes can be ruled out if they are reduced by the survey design. Which then are mode-inherent characteristics that create the mode effect and cannot be avoided even with a clever survey design?

While different authors define mode-inherent characteristics and hence differences between the modes in their own terms, mode-specific characteristics may broadly be defined as a combination of the interviewer's involvement, the degree of contact with the respondent, the channel of communication, the media of communication, the locus of control, degree of privacy and the presence of computer technology ${ }^{7}$ (Berzelak, 2014; Couper, 2011; Groves et al. 2009; de Leeuw, 1992). Authors list such characteristics as modeinherent properties that create mode effects and usually cannot be avoided by a clever survey design or sampling (Berzelak, 2014). While this article cannot fully review all of them or provide an overall single view on them, we shall briefly examine four of the characteristics we believe are typically given more thought when designing survey ${ }^{8}$ and relate each of them to context effects.

Involvement of the interviewer is certainly the most obvious and generally agreed mode characteristic (Couper, 2011; Groves, et al., 2009; de Leeuw, 1992; Tourangeau, Rips, and Rasinski, 2000).

On the positive side, the interviewer can provide a controlled environment for the interview by arranging the time and (with a face-to-face survey) location of the interview so it can be conducted with the fewest interruptions possible - in contextual terms, he can ensure the most appropriate social context for the interview (Bradburn, 1991). By providing the most appropriate social context, the interviewer can consequently influence the local context within which the interview is carried out.

The interviewer also relieves the respondent of the cognition burden induced by the questionnaire by taking the locus of control into his domain (for example, by taking care of the routing in the questionnaire) or even talking to less literate respondents who would struggle to read the questionnaire. Trained interviewers can also motivate and engage the respondent (Callegaro, Lozar Manfreda, and Vehovar, 2015). Both respondent capability and respondent motivation are mentioned in the previous section and fall within the local context as defined by Uhan (1998). Consequently, the mode effect due to the interviewer's presence can moderate the context regarding respondents capability and motivation when understood in terms of Bradburn (1991) and Uhan (1998).

Despite being a professional, an interviewer can sometimes influence a respondent

${ }^{7}$ For a more in-depth overview of mode characteristics, see Berzelak (2014), pp. 32-36.

${ }^{8}$ E.g. by deciding on the interviewer involvement, use of computer or/and showcards, etc. 
via conversational implicatures (see the section above). This is a global context effect which is only present in survey modes that use an interviewer. Some respondents feel the interviewer is a representative of authority (Uhan, 1998). Both the traditional and contemporary psychological theory of social influence explain the processes occurring in the interviewer's presence, such as compliance and social desirability. First, Kelman's (1958) compliance social influence process ${ }^{9}$ can clearly be identified by the interviewer's presence. Compliance means a respondent provides a particular answer "to achieve favorable reaction from another person a group" (Kelman 1958, p. 3). More recently, Friedkin (1998) built upon the theory of social influence by adding a structural approach to it. In addition, Tourangeau and Yan (2007) note that social desirability is quite common in surveys (Tourangeau and Yan, 2007). Being influenced by the presence of the interviewer or a third party on one hand and the sense of privacy in self-administered data collection modes on the other, social desirability is widely accepted as a mode-inherent characteristic (Callegaro, Lozar Manfreda, and Vehovar, 2015; Tourangeau and Yan, 2007; Couper, 2011; Dillman and Christian, 2005). While social desirability is indeed a mode-inherent effect, Bradburn (1991) also suggests that it is part of the social context in which the survey interview is conducted.

Based on the above arguments, we propose that interviewer presence (involvement) is a moderator of the survey context and thus of the context effects.

The presence of computer technology is identified as a mode-inherent characteristic by different authors (Couper, 2011; de Leeuw, 2005; Berzelak, 2014). Computer presence used to have (and therefore can still have) an impact on the data even when used solely by the interviewer because some respondents fear that computers are monitoring the consistency of their answers (de Leeuw, 1992). While later sources disregard this phenomenon as today being almost negligible (Snijkers, Hox, and de Leeuw, 1995; de Leeuw, 2002), the presence of a computer may still change the relations and social setting, thereby affecting the social context.

Another computer-related effect that can only be observed when respondents actually have to use it is the level of acquaintance with computer technology (Callegaro, Lozar Manfreda, and Vehovar, 2015). When a computer is used in telephone surveys, only the locus of control changes from the interviewer to the computer - the respondent neither sees nor uses the device but the interviewer's work is made easier so they can be more concentrated on motivating the respondent.

Consequently, we find that the computer forms part of the social setting in which the interview is carried out. When used by the respondent (compared to a paper-based questionnaire), the computer may take the locus of control as well as engage and motivate the respondent through visual design, interactivity and other elements (Callegaro, Lozar Manfreda, and Vehovar, 2015). As the presence of computer technology influences the social setting which is part of the [social] context, it also influences the context effects.

Another mode-inherent characteristic is the channel of communication (Groves, et al., 2009; Berzelak, 2014). It can be aural (telephone), visual (paper or web questionnaire) or a combination of both (face-to-face interview). Tourangeau, Rips and Rasinski go even further and distinguish channels of presentation and responding (Tourangeau, Rips, and

\footnotetext{
${ }^{9}$ Kelman identified three social influence processes: compliance, identification and internalisation (Kelman, 1958).
} 
Rasinski, 2000). An example of an interview involving different channels of presentation and responding is IVR where the respondent listens to the question and then enters the appropriate answer on the telephone keypad. The traditional literature discusses the dichotomy of oral and literal communication whereby written language is more analytic, classificatory and structured while oral is redundant and aggregative (Ong, 1982). Murray (1988) systematises this dichotomy as emotional, personal, context-bound oral communication vs. analytic and sequential that is suitable for rational and critical thinking written text. He (Murray, 1988) further experimented with the computer as the medium of data collection and concluded that mode and medium represent choices made due to the characteristics of the particular context in which the interaction takes place (Murray, 1988). While demonstrating that literacy and orality are not dichotomous, as otherwise suggested by the traditional literature, he placed channel as well as medium of communication (described here as mode-inherent characteristics) directly in the context of the interaction (Murray, 1988).

In addition, de Leeuw, Hox, and Dillman (2008) suggested that social conventions, also defined as global context effects by Uhan (1998), can be shaped by the channel of communication.

Medium of communication is the last mode-inherent characteristic we use to demonstrate that context effects are moderated by the mode of data collection. Primarily influenced by Tourangeau and de Leeuw, Berzelak defined it as an information transmission medium, which is "the tool (or service) used to transfer questionnaire and answers between the respondent and the researcher" (Berzelak, 2014, p. 34). He further explains that it can be "in person, via telephone line, mail or other form of physical delivery, email, or web" (Berzelak, 2014, p. 34). Returning to his sources, we find the definition of medium of communication given by de Leeuw (2005) to be one of the most complete and context-aware. She defines medium-related factors as "social conventions and customs associated with the media utilized in the survey methods, such as familiarity with a medium, use of medium and locus of control" (de Leeuw, 2005, p. 244).

This definition is literally written in terms of context effects by explicitly mentioning "social conventions and customs", similarly to Bradburn (1991) and Uhan (1998). In our opinion, this definition is another formulation of the global contextual view on the interview process. As we understand it, she meant that the use of any medium per se can cause a certain traces on the data as social conventions and customs vary among different media of communication. Therefore, when the medium of communication is changed, the global context with all the related conventions and customs also changes.

Selected mode-inherent characteristics, their (alleged) impact on the survey data as well as their relationship to context effects as described above are summarised in Table 2 below.

Let us give an empirical example to illustrate our notion of mode being a moderator of context. It can be seen from the experience from the European Social Survey (ESS) ${ }^{10}$ and the European Values Study (EVS): ${ }^{11}$ in order to counteract the differences created by changing the mode from face to face (F2F) to web, in ESS they had to adapt $85 \%$

\footnotetext{
${ }^{10}$ European Social Survey is a repeat cross-sectional survey on attitudes, beliefs and behavior that is conducted in over 30 countrier (ESS, 2017).

${ }^{11}$ European Values Study is a repeated cross-section survey on basic human values that is conducted in over 45 countries (EVS, 2017).
} 
Table 2: Systematisation of selected mode-inherent characteristics, their (alleged) impact on the survey data and relationship to context effects

\begin{tabular}{|c|c|c|}
\hline $\begin{array}{l}\text { Mode- } \\
\text { inherent } \\
\text { characteristics }\end{array}$ & Key possible impacts on data & Related context effects \\
\hline $\begin{array}{l}\text { Presence of } \\
\text { the inter- } \\
\text { viewer (degree } \\
\text { of interviewer } \\
\text { involvement) }\end{array}$ & $\begin{array}{l}\text { - provoke socially desirable an- } \\
\text { swers } \\
\text { - cause interviewer bias } \\
\text { - relieve respondent of cognitive } \\
\text { burden } \\
\text { - provide the appropriate envi- } \\
\text { ronment } \\
\text { - motivate the respondent }\end{array}$ & $\begin{array}{l}\text { Local context: Frame the social } \\
\text { context of the interview } \\
\text { Global context: Bring inter- } \\
\text { viewer's personality traits, beliefs } \\
\text { and experience into the interview }\end{array}$ \\
\hline $\begin{array}{l}\text { Channel of } \\
\text { communica- } \\
\text { tion (aural vs. } \\
\text { visual) }\end{array}$ & $\begin{array}{l}\text { - provide access to the illiterate } \\
\text { (aural) provide access to the } \\
\text { deaf (visual) } \\
\text { - ease the survey response pro- } \\
\text { cess for some individuals (de- } \\
\text { pending on their characteris- } \\
\text { tics) }\end{array}$ & $\begin{array}{l}\text { Local context: Trigger different } \\
\text { conversational implicatures }\end{array}$ \\
\hline $\begin{array}{l}\text { Presence of } \\
\text { computer } \\
\text { technology }\end{array}$ & $\begin{array}{l}\text { - limit (or provide) access to cer- } \\
\text { tain target groups } \\
\text { - instigate different social con- } \\
\text { ventions } \\
\text { - reduce random errors due to } \\
\text { locus of control } \\
\text { - demonstrate social segmenta- } \\
\text { tion (access to the computer, } \\
\text { acquaintance) }\end{array}$ & $\begin{array}{l}\text { Local context: Alter the social } \\
\text { setting during the interview } \\
\text { Global context Bias respondent's } \\
\text { profile by segmenting regarding } \\
\text { access to computer and acquain- } \\
\text { tance with the computer }\end{array}$ \\
\hline $\begin{array}{l}\text { Medium of } \\
\text { communication }^{\mathrm{a}}\end{array}$ & $\begin{array}{l}\text { - limit (or provide) access to cer- } \\
\text { tain target groups } \\
\text { - trigger different social conven- } \\
\text { tions, reduce (increase) ran- } \\
\text { dom errors due to locus of con- } \\
\text { trol } \\
\text { - demonstrate social segmenta- } \\
\text { tion (access to the medium) }\end{array}$ & $\begin{array}{l}\text { Local context: Provoke medium- } \\
\text { specific social conventions and } \\
\text { customs } \\
\text { Global context Bias respondent's } \\
\text { profile by segmenting regarding } \\
\text { medium access }\end{array}$ \\
\hline
\end{tabular}

a As proposed by Berzelak (2014) based on Tougangeau and de Leeuw's texts. 
of the questions while in EVS they changed the wording of 156 out of 256 questions (Callegaro, Lozar Manfreda, and Vehovar, 2015) to ensure that the equivalence of the data was retained. We find this change in line with Ong's (1982) dichotomy of classificatory and structured written vs. redundant and aggregative oral communication and even more so with Goody and Watt's (1963) explanation of semantic ratification, by which "the meaning of each word is ratified in a succession of concrete situations, accompanied by vocal inflexions and physical gestures, all of which combine to particularize both its specific denotation and its accepted connotative usages" (Goody and Watt, 1963, p. 306).

Both the ESS and EVS empirical evidence as well as Goody and Watt (1963) demonstrate that an intra-survey context adjustment (adaptation of the questionnaire) can provide (approximately) equivalent resulting data when changing the data collection mode from F2F to web. As shown in Table 2, mode-inherent features can have an influence on extrasurvey context effects. Because the change of mode altered the extra-survey context and this effect can be reduced by altering the intra-survey context (questionnaire), we suggest that mode itself is a moderator of context and therefore of context effects.

\section{Why it Matters}

The above discussion demonstrates that the mode of data collection inherently affects the context effects within which the survey interview takes place. From this viewpoint, researchers could adjust the survey design to mitigate certain local context effects while explaining some of the "difficult to predict" (Martin and Lynn, 2011, p. 30) effects in terms of Uhan's global context. For illustrative purpose, consider two scenarios of crossnational surveys being conducted in the same countries:

1. a completely standardised (coherent sampling, coordinated translations, single mode etc.) cross-national comparative survey (such as the European Social Survey); and

2. a less standardised cross-national comparative survey (such as the International Social Survey Programme).

While global context cannot be directly controlled for, it can be coherent among similar countries that have similar respondent sampling frames and interviewer bases. However, it can also vary quite considerably if the countries involved are sufficiently different. In the first scenario, the completely standardised design provides the same canvas (local context effect) while the local context effect in the second example may change due to inequivalent translations, the mode change etc. In the second scenario, the countries involved are quite similar (very similar global context) while the local context is different due to the less rigorous survey design standard.

Both local and global contexts as well as the two scenarios are visualised in Picture 1.

If the global contexts vary substantially ${ }^{12}$ (such as in scenario 1 in Figure 1), providing the same local context may be futile since irrespective of how similar the local context is, the results are considerably inequivalent due to the different global contexts. Scenario 2 demonstrates the inverse situation: very similar global contexts provide the foundation for

\footnotetext{
${ }^{12}$ E.g.: if the countries involved have different cultural, religious or political value systems.
} 


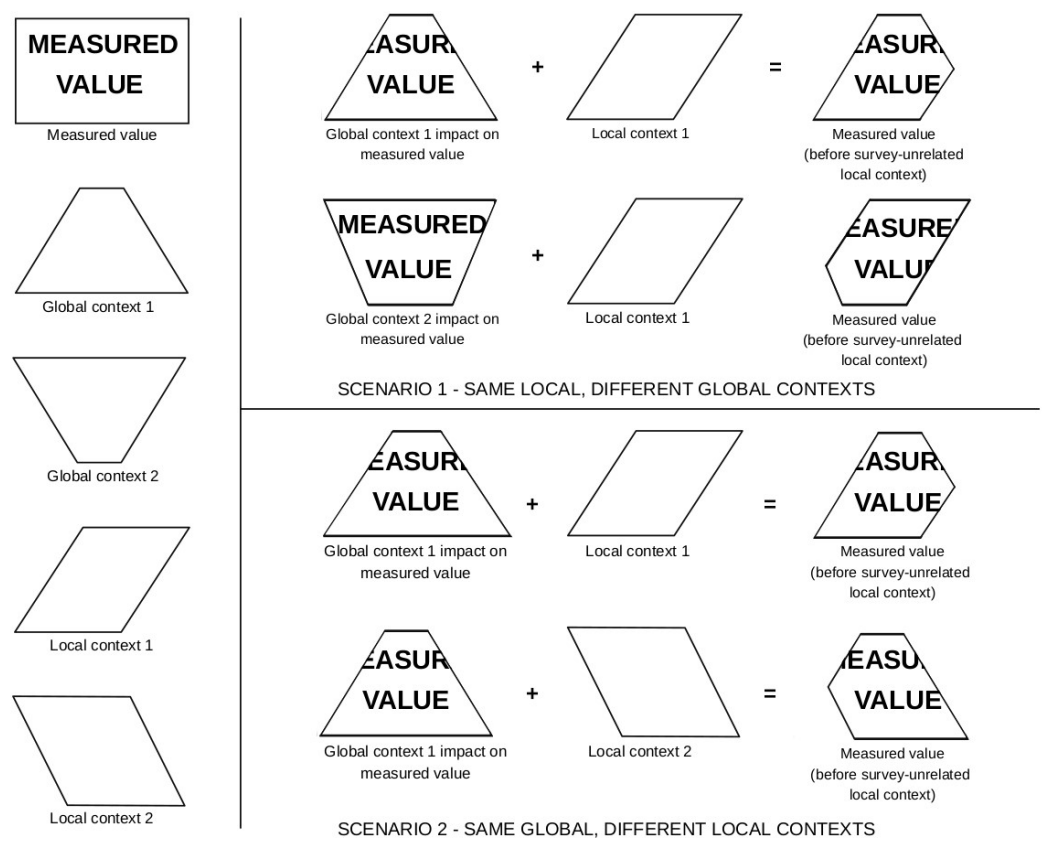

Figure 1: Comparison of the two survey scenarios with the same local or same global context effects

higher data equivalence but the opportunity is missed due to different local context introduced by the less rigorous survey design specifications. The two cases produce different measured values.

The depiction of the two scenarios emphasizes the true importance of the survey design for the data obtained. While the amount and impact of both contexts cannot be assessed beforehand without experimentation, based on the two above scenarios we argue that, when the global context has a substantially greater effect on the data than the local context (which includes mode), any effort towards rigid standardization such as in scenario 1 may be futile.

As this view can only be valid when mode of data collection is a moderator of context, we find this relationship important.

\section{Conclusion}

Based on both empirical and theoretical evidence, we demonstrated that the effects of data collection mode may not be separate phenomena but an integral moderating part of context effects. This view could provide the basis for new and more effective techniques to be developed to ensure data comparability in mixed-mode comparative surveys and optimal use of resources where some standardisation procedures would prove ineffective.

There are many questions in need of answers so further empirical evidence and experimentation is required in order to assess the degree of moderation and the relationship between different modes within standardised contexts before such techniques can be developed.

Until then, we should take Martin and Lynn (2011) into account who urged caution 
when comparing data obtained using different modes.

\section{References}

[1] Adorno, T., Frenkel-Brunswik, E., Levinson, D., and Sanford, R. (1950): The Authoritarian Personality. New York: Harper.

[2] Berzelak, J. (2014): Mode Effects in Web Surveys. Ph.D thesis of University of Ljubljana, Ljubljana.

[3] Bingham, W., and Moore, B. (1934): How to Interview. New York: Harper and Row.

[4] Bradburn, N. M. (1991): What have we learned? In N. Schwarz, and S. Sudman (Eds.): Context Effects in Social and Psychological Research, 315-323. New York: Springer-Verlag,

[5] Callegaro, M., Lozar Manfreda, K., and Vehovar, V. (2015): Web Survey Methodology. London: Sage.

[6] Campbell, A., Converse, P., Miller, W., and Stokes, D. (1960): The American Voter. New York: Wiley.

[7] Cannell, C. and Fowler, F. (1963): Comparison of a self-enumerative procedure and a personal interview: A validity study. Public Opinion Quarterly, 27(2), 250-264.

[8] Chang, L. and Krosnick, J. (2010): Comparing oral interviewing with selfadministered computerized questionnaires: An experiment. Public Opinion Quarterly, 74(1), 154-167.

[9] Couch, A. and Keniston, K. (1960): Yeasayers and naysayers: Agreeing response set as a personality variable. The Journal of Abnormal and Social Psychology, 60(2), $151-174$

[10] Couper, M. (2011): The future of modes of data collection. Public Opinion Quarterly, 75(5), 889-908.

[11] de Heer, W., de Leeuw, E. D., and van der Zouwen, J. (1999): Metholological issues in survey research: A historical review. Bulletin of Sociological Methodology/Bulletin de Méthodologie Sociologique, 64(1), 25-48.

[12] de Leeuw, E. (1992): Data Quality in Mail, Telephone and Face-to-Face Surveys. Amsterdam: TT-Publikaties.

[13] de Leeuw, E. (2002): The effect of computer-assisted interviewing on data quality: A review of the evidence. In J. Blasius, J. Hox, E. D. de Leeuw, and P. Schmidt (Eds.): Social Science Methodology in the New Millennium. Opladen: Leske + Budrich.

[14] de Leeuw, E. (2005): To mix or not to mix data collection modes in surveys. Journal of Official Statistics, 21(2), 233-255. 
[15] de Leeuw, E. and Berzelak, N. (2016): Survey mode or survey modes? In C. Wolf, D. Joye, T. W. Smith and Y. Fu (Eds.): The SAGE Handbook of Survey Methodology, 142-156. London: Sage

[16] de Leeuw, E., Hox, J., and Dillman, D. (2008): International Handbook of Survey Methodology. New York: Taylor and Francis Group.

[17] DeMaio, T. J. (1985): Social desirability and survey measurement: A review. In C. Turner, and E. Martin (Eds.): Surveying Subjective Phenomena 2, 257-282. New York: Sage.

[18] Deming, W. (1944): On errors in surveys. American Sociological Review, 9(4), 359369.

[19] Dillman, D., and Christian, L. (2005): Survey mode as a source of instability in responses across surveys. Field Methods, 17(1), 30-52.

[20] Dillman, D., Phelps, G., Tortora, R., Swift, K., Kohrell, J., Berck, J., and Messer, B. (2009): Response rate and measurement differences in mixed mode surveys using mail, telephone, Interactive Voice Response (IVR) and the Internet. Social Science Research, 1-18.

[21] ESS. (2017): About the European Social Survey European Research Infrastructure - ESS ERIC. Downloaded 04. Sep 2017 from European Social Survey: http://www.europeansocialsurvey.org/about/

[22] EVS. (2017): About EVS. Downloaded 04. Sep 2017 from European Values Study: http://www.europeanvaluesstudy.eu/page/about-evs.html

[23] Friedkin, N. E. (1998): A Structural Theory of Social Influence. Cambridge: Cambridge University Press.

[24] Goody, J., Watt I. (1963): The consequences of literacy. Comparative Studies in Society and History. 5(3), 304-345

[25] Grice, H. (1974): Logic and conversation. In P. Cole, and L. Morgan (Eds.): Syntax and Semantics 3: Speech Arts, 44-58. New York: Elsevier.

[26] Groves, R., Fowler, F., Couper, M., Lepkowski, J., Singer, E., and Tourangeau, R. (2009): Survey Methodology. Hoboken, NJ: John Wiley and Sons.

[27] Hafner-Fink, M., and Uhan, S. (2013): Bipolarity and/or duality of social survey measurement scales and the question-order effect. Quality \& Quantity, 47(2), 839852.

[28] Hox, J., de Leeuw, E., and Zijlmans, E. (2015): Measurement equivalence in mixed mode surveys. Frontiers in Psychology, 6, 1-11.

[29] ISSP. (2010): General information. Downloaded 28. Sep 2016 from International Social Survey Programme: http://www.issp.org/index.php 
[30] Jäckle, A., Roberts, C., and Lynn, P. (2010): Assessing the effect of data collection mode on measurement. International Statistical Review, 78(1), 3-20.

[31] Kaplowitz, M., Hadlock, T., and Levine, R. (2004): A comparison of Web and mail response rates. Public Opinion Quarterly, 68(1), 94-101.

[32] Karol, J. (1937): Measuring radio audiences. The Public Opinion Quarterly, 1(2), 92-96.

[33] Krosnick, J. (1991): Response strategies for coping with the cognitive demands of attitude measures in surveys. Applied Cognitive Psychology, 5(3), 213-236.

[34] Krosnick, J. (1999): Survey research. Annual Review of Psychology, 50(1), 537567.

[35] Kelman H. C. (1958): Compliance, identification and internalization three processes of attitude change. Conflict Resolution, 2(1), 51-60.

[36] Lavrakas, P. (2008): Encyclopedia of Survey Research Methods. London: Sage.

[37] Lozar Manfreda, K., and Vehovar, V. (2002): Do mail and Web surveys provide same results? Metodološki zvezki, 18, 149-169.

[38] Martin, P., and Lynn, P. (2011): The Effects of Mixed Mode Survey Designs on Simple and Complex Analyses. London: City University of London.

[39] Mayer-Schönberger, V., and Cukier, K. (2013): Big data: A Revolution That Will Transform How We Live, Work and Think. New York: Houghton Mifflin.

[40] Murray, D. E. (1988): The context of oral and written language: A framework for mode and medium switching. Language in Society, 17(3), 351-373.

[41] Ong, W. J. (1977): Interfaces of the World: Studies in the Evolution of Consciousness and Culture. London: Cornell University Press.

[42] Roberts, C. (2007): Mixing Modes of Data Collection in Surveys: A Methodological Review. London: ESRC National Centre for Research Methods.

[43] Roxas, B., and Lindsay, V. (2012): Social desirability bias in survey research on sustainable development in small firms: An exploratory analysis of survey mode effect. Business Strategy and the Environment, 21(4), 223-235.

[44] Sakshaug, J. W., Yan, T., and Tourangeau, R. (2010): Nonresponse error, measurement error, and mode of data collection: Tradeoffs in a multi-mode survey of sensitive and non-sensitive items. Public Opinion Quarterly, 74(5), 907-933.

[45] Sax, L., Gilmartin, S., and Bryant, A. (2003): Assessing response rates and nonresponse bias in Web and paper surveys. Research in Higher Education, 44(4), 409432. 
[46] Schuman, H., and Presser, S. (1996): Questions and Answers in Attitude Surveys: Experiments on Question Form, Wording and Context. London: Sage.

[47] Schwarz, N., and Strack, F. (1991): Context effects in attitude surveys: Applying cognitive theory to social research. European Review of Social Psychology, 31-50.

[48] Schwarz, N., and Sudman, S. (1991): Context Effects in Social and Psychological Research. New York: Springer-Verlag.

[49] Snijkers, G., Hox, J., and de Leeuw, E. (1995): The effect of computer-assisted interviewing on data quality. A review. International Journal of Market Research, 37(4), 325-344.

[50] Tourangeau, R., and Rasinski, K. (1988): Cognitive processes underlying context effects in attitude measurement. Psychological Bulletin, 103(3), 299-314.

[51] Tourangeau, R., and Yan, T. (2007): Sensitive questions in surveys. Psychological Bulletin, 133(5), 859-883.

[52] Tourangeau, R., Rips, L., and Rasinski, K. (2000): The Psychology of Survey Response. Cambridge: Cambridge University Press.

[53] Uhan, S. (1998): Prava in neprava mnenja. Ljubljana: Fakulteta za družbene vede.

[54] Vannieuwunhuyze, J., Loosveldt, G., and Molenberghs, G. (2010): A method for evaluating mode effects in mixed-mode surveys. Public Opinion Quarterly, 74(5), 1027-1045. 